\title{
Microglia limit the expansion of $\beta$-amyloid plaques in a mouse model of Alzheimer's disease
}

Ruohe Zhao ${ }^{1,2}$, Wanling Hu${ }^{1}$, Julia Tsai ${ }^{2}$, Wei Li ${ }^{1,2^{*}}$ and Wen-Biao Gan ${ }^{1,2^{*}}$

\begin{abstract}
Background: Microglia are known as resident immune cells in the brain. $\beta$-amyloid (A $\beta$ ) plaques in the brain of Alzheimer's disease (AD) are surrounded by microglia, but whether and how microglia affect the formation and maintenance of plaques remains controversial.

Methods: We depleted microglia by injecting diphtheria toxin (DT) in $C X_{3} C R 1^{C r E E R /+}: R 26^{D T R /+}\left(C X_{3} C R 1\right.$-iDTR) mice crossed with APPSwe/PSEN1dE9 (APP/PS1) mice. Intravital time-lapse imaging was performed to examine changes in the number and size of Congo Red-labeled amyloid plaques over 1-2 weeks. We also examined spine density and shaft diameter of dendrites passing through plaques in a PSAPP mouse model of AD (PS1 M146L line $6.2 \times \mathrm{Tg}_{2576}$ ) crossed with Thy1 YFP H-line mice.

Results: We found that DT administration to $C X_{3} C R 1$-iDTR mice efficiently ablated microglia within one week and that microglia repopulated in the second week after DT administration. Microglia depletion didn't affect the number of amyloid plaques, but led to $\sim 13 \%$ increase in the size of A $\beta$ plaques within one week. Moreover, microglia repopulation was associated with the stabilization of plaque size during the second week. In addition, we found dendritic spine loss and shaft atrophy in the distal parts of dendrites passing through plaques.
\end{abstract}

Conclusion: Our results demonstrate the important role of microglia in limiting the growth of $A \beta$ plaques and plaque-associated disruption of neuronal connection.

Keywords: Alzheimer's disease, A $\beta$ plaque, Microglia depletion, Two-photon imaging, APP/PS1, CX ${ }_{3} C R 1^{\mathrm{CreER} /+}: \mathrm{R}_{2} 6^{\mathrm{DTR} /+}$

\section{Background}

Accumulation of $\beta$-amyloid $(A \beta)$ in the brain is a hallmark of Alzheimer's disease (AD) [1, 2]. Studies of postmortem $\mathrm{AD}$ brains have revealed dystrophic neurites inside and surrounding $A \beta$ plaques [3-6]. Studies in AD mouse models have also shown that $A \beta$ deposition is associated with various neuronal abnormalities, including the formation of dystrophic neurites $[5,7,8]$, dendritic spine loss $[5,7,9,10]$, synaptic dysfunction $[11,12]$ and abnormal neuronal firing $[13,14]$. The variety of neuronal deficits associated with $A \beta$ deposition likely contribute to memory loss and cognitive decline in $\mathrm{AD}$ patients [15-19].

\footnotetext{
*Correspondence: liwei@pkusz.edu.cn; gan@saturn.med.nyu.edu ${ }^{1}$ Drug Discovery Center, Peking University Shenzhen Graduate School, Shenzhen 518055, China

Full list of author information is available at the end of the article
}

Microglia are the resident immune cells in the central nervous system [20,21]. They are clustered around amyloid plaques in both human $\mathrm{AD}$ brains $[22,23]$ and the brains of AD mouse models [24, 25]. Mutations in genes such as CD33 and TREM2 have been linked to microglia dysfunction and increased risks for AD [26-28]. Knockout of $C X_{3} C R 1$ [29, 30], CD33 [27], or TREM2 [31, 32] leads to altered plaque load, suggesting that microglia are involved in $A \beta$ deposition. Activated microglia have been shown to phagocytose $A \beta$ through scavenger receptormediated mechanisms [33-35]. Furthermore, in vivo imaging studies have shown that microglia volume surrounding plaques correlated with the decrease of plaque size [25]. Microglia processes enveloping plaque surface are suggested to constitute a barrier to prevent plaque expansion [36, 37], and the disruption of this barrier in TREM2 deficiency mice is associated with the increase of 
dystrophic neurites [32, 37]. Together, these studies lead to a view that microglia are involved in the clearance of $A \beta$ and limiting the expansion of plaques.

Although many lines of evidence suggest a role of microglia in restricting the growth of amyloid plaques, several studies have shown that microglia depletion does not affect the number and size of plaques in mouse models of $\mathrm{AD}$. It has been reported that chronic microglia depletion for one month by activating suicide gene $H S V T K$ under $C D 11 b$ promoter has no effects on the formation or maintenance of plaques [38]. In another study, microglia are depleted by inhibiting colonystimulating factor 1 receptor (CSF1R) with PLX3397 from Plexxikon. PLX3397 treatment for 4 weeks results in nearly complete depletion of microglia, but has no effects on plaque load in a mouse model of AD [39]. Thus, in contrast to the view that microglia are beneficial to limiting amyloid deposits, these depletion studies suggest that microglia have no significant effect on the formation and growth of amyloid plaques.

To better understand the role of microglia in $A \beta$ deposition, we examined the number and size of plaques after depleting microglia by administration of diphtheria toxin (DT) to $C X_{3} C R 1^{\mathrm{CreER} /+}: \mathrm{R}^{2} 6^{\mathrm{DTR} /+}\left(\mathrm{CX} \mathrm{X}_{3} \mathrm{CR} 1-i D T R\right)$ mice crossed with APPswe/PSEN1dE9 (APP/PS1) mice. Using two-photon time-lapse imaging of $A \beta$ plaques in mice more than 12 months old, we found that $A \beta$ plaque size, but not plaque number, showed a significant increase over 7 days after microglia depletion. In addition, we found a significant reduction in dendritic spine density and dendritic diameter in the distal segments of dendrites passing through plaques. Taken together, our results suggest that microglia play an important role in limiting the growth of $A \beta$ plaques and reducing neuronal damage associated with $A \beta$ deposition.

\section{Methods}

Transgenic mice

APPswe/PSEN1dE9 (APP/PS1) were purchased from Guangdong Medical Laboratory Animal Center, China. C57BL/6 mice expressing YFP in layer-V pyramidal neurons (Thy1 H-line) and Rosa26-stop-iDTR (DTR) mice were purchased from the Jackson Laboratories, US. $\mathrm{CX}_{3} \mathrm{CR} 1^{\mathrm{CreER}}$ mice were generated in New York University School of Medicine [40]. These mice were crossbred and litters were genotyped by PCR using the following primers: for APP/PS1 mice, 5' - TCATGACTATCCT CCTGGTGG-3' and 5' $^{\prime}$ - CGTTATAGGTTTTAA ACACTTCCCC-3'; for $C X_{3} C R 1^{\text {CreER }}$ mice, 5' - AAGAC TCACGTGGACCTGCT-3', 5' - CGGTTATTCAACTT GCACCA-3' and 5' ${ }^{\prime}$ AGGATGTTG ACTTCCG AGTTG-3'; for DTR mice, 5' - CTGGCTTCTGAGGACCG-3' and 5'-CGAAGAGTTTGTCCTCAACCG-3'. 12-24 months old quadruple transgenic mice were used for microglia depletion experiment and in vivo imaging. Age-matched animals with APP/PS1 mutation but no CreER or DTR gene were used as controls.

For studies of dendritic abnormalities near plaques, mutant human amyloid precursor protein (FAD $A P P_{670 /}$ 671 line $\mathrm{Tg} 2576)$ and mutant human Presenilin 1 (PS1 $1_{\mathrm{M} 146 \mathrm{~L}}$ line 6.2) mice were obtained from Taconic and the University of South Florida, respectively. All experiments were done in accordance with the institutional guidelines.

\section{Labeling amyloid plaques in vivo}

Mice were anesthetized with pentobarbital sodium (100 mg/kg) 24-48 h before imaging. Congo Red was injected into the subarachnoid space using a fine glass electrode guided by a micromanipulator. The electrode was backfilled with Congo Red ( $3 \mu \mathrm{l}, 0.5 \%$ in artificial cerebral spinal fluid (ACSF), filtered through a $0.20 \mu \mathrm{m}$ syringe filter before use, Sigma) and inserted through the thinned skull. After subarachnoid space was reached (as evidenced by free dye diffusion), Congo Red was then pressure injected with a picospritzer $(20$ p.s.i., $20 \mathrm{~ms}$, $0.8 \mathrm{~Hz}$ ) over 20-30 min. Dye labeling of amyloid plaques was typically observed within $30 \mathrm{~min}$ of injection.

\section{In vivo two-photon imaging of $A \beta$ plaques}

The procedure of transcranial two-photon imaging was described previously [7, 41]. Briefly, after exposing the skull surface and gluing the skull to a custom-made steel plate, a small region $\sim 1 \mathrm{~mm}$ in diameter was thinned with a highspeed drill, and a microsurgical blade was then used to continue thinning until the skull area $\sim 500 \mu \mathrm{m}$ in diameter was $\sim 20 \mu \mathrm{m}$ in thickness. A picture of the brain vasculature in the thinned region was taken with a $C C D$ camera and used as a landmark for future relocation. Animals were placed under a two-photon microscope, and image stacks of plaques within a depth of $300 \mu \mathrm{m}$ from the pial surface were obtained in the step size of $1 \mu \mathrm{m}$ with a 1.05 N.A. $25 \times$ water-immersion objective and with two-photon laser tuned to $880 \mathrm{~nm}$. For each frame, a $512 \times 512$ pixel, zoom $1 \times$ image was taken from a $508 \times 508 \mu \mathrm{m}$ ROI. Image stacks from motor, somatosensory and visual cortices yielded a full three-dimensional data set of $A \beta$ plaques labeled with Congo Red in these cortical regions. After imaging, the plate was gently detached from the skull and the scalp was sutured, and the animals were returned to their home cages until the next viewing.

\section{Microglia depletion}

Depletion of microglia was performed according to previously published studies [40]. Animals received two doses of $10 \mathrm{mg}$ of tamoxifen $(20 \mathrm{mg} / \mathrm{ml}$, dissolved in corn oil, Sigma) to induce Cre-mediated recombination and DTR 
expression, with a separation of $48 \mathrm{~h}$ between doses. Diphtheria toxin (Sigma) was diluted in PBS $(50 \mu \mathrm{g} / \mathrm{ml})$ and $1 \mu \mathrm{g}$ of toxin was given i.p. for three consecutive days for depletion of microglia.

\section{Immunohistochemistry}

Mice were anesthetized and perfused with $0.9 \%$ PBS. CNS tissue was removed and fixed in 4\% PFA, rinsed with PBS and sectioned at $150 \mu \mathrm{m}$ with a vibratome. Sections were permeabilized in 1\% Titron X-100 in PBS for $3 \mathrm{~h}$ and blocked with $5 \%$ normal goat serum for $1 \mathrm{~h}$. Sections were incubated overnight with primary antibodies against Iba1 (Wako, 1:500). Sections were then washed with PBS/ $0.05 \%$ Tween-20, and then incubated with Alexafluorconjugated goat anti-rabbit IgG secondary antibodies (Life Technologies, 1:500) for $2 \mathrm{~h}$. Sections were washed as before and mounted for imaging. Confocal images were obtained on a Biorad Radiance 2000 confocal microscope.

\section{Labeling and imaging fixed brain slice}

Transversal slices (150-200 $\mu \mathrm{m}$ thick) of fixed brains were cut on a vibratome. Congo Red was used to visualized fibrillary amyloid in triple $Y F P / A P P / P S 1$ transgenic mice. Fixed brain slices were incubated in $0.5 \%$ Congo Red for $30 \mathrm{~min}$ and then rinsed with $0.1 \mathrm{M}$ PBS. Slices were then mounted between two glass cover-slips in Vectashield (Vector Laboratories) and sealed with dental wax.

Labeled brain slices were imaged by laser scanning confocal microscopy using either a N.A. $1.2540 \times$ or a N.A. $1.360 \times$ oil-immersion objective. Neuronal structures labeled with YFP and fibrillar amyloid deposits with Congo Red were scanned sequentially using $488 \mathrm{~nm}$ and $568 \mathrm{~nm}$ laser excitation, respectively. Image stacks at $0.3-1.0 \mu \mathrm{m}$ steps were acquired to generate three-dimensional data sets of neuronal structures and amyloid plaques.

\section{Image analysis}

To analyze the in vivo images of $A \beta$ plaques, all images were processed using a custom-written Matlab algorithm. For each plaque, the frame with highest mean fluorescence intensity out of the entire image stack was identified as the center frame of this plaque. 2 additional frames above and below the center (5 frames combined) were used to analyze plaque size. In each image frame, plaque border was determined by setting a threshold of 3 times of standard deviation of the background fluorescence (mean value of the lowest $10 \%$ fluorescence intensity of the entire image) of the current frame. The averaged plaque area with the border was used as the plaque size.

To quantify fluorescence surrounding a plaque, fluorescence intensity across a radial line from the center point towards the outer area of a plaque was extracted. This radial line was drawn in a randomized direction and was kept in the same direction in analyzing the same plaque between different imaging sessions. This radial line was separated into the "inside" part and the "outside" part by the border of plaque. Fluorescence intensity across the line was normalized to a $0-100 \%$ scale with the highest value across the line as $100 \%$. Normalized fluorescence intensity within $5 \mu \mathrm{m}$ surrounding plaque border in the "outside" part was used as the measurement of fluorescence intensity surrounding the plaque.

To quantify changes of dendritic structures near $\mathrm{A} \beta$ deposits in fixed brain slices, dendritic spine densities and shaft diameters were measured with Metamorph software. Spine density and shaft diameter were measured for the proximal or distal dendritic segment (proximal or distal dendritic segments had comparable length, ranging from 20 to $80 \mu \mathrm{m}$ ), depending on the region in which the segment was located. Segments close to the soma and before the plaques were defined as proximal segments. Segments far away from the soma and after plaques were defined as distal segments. The border of $\mathrm{A} \beta$ deposits was determined as the position in which a sharp increase in fluorescence intensity was observed.

\section{Statistics}

All data were presented as mean \pm S.E.M. KolmogorovSmirnov test was used to test normality of sample's distribution. Student's t-test (two-tailed) was used to test for differences between groups whose distributions passed Kolmogorov-Smirnov test. Independent-samples MannWhitney test and related-samples Wilcoxon Signed rank test was used to compare differences between groups whose distributions did not pass Kolmogorov-Smirnov test. Significant levels were set at $P \leq 0.05$. All statistical analyses were performed using the IBM SPSS Statistics 23.

\section{Result}

Cre-dependent microglia depletion in the brain

Previous studies have reported that microglia depletion by activating suicide gene HSVTK or by inhibiting CSFR1 had no significant effect on the number and size of amyloid plaques in mouse models of $\mathrm{AD}[38,39]$. To better understand the role of microglia in amyloid deposition, we took advantage of a recently-developed method to deplete microglia by administrating diphtheria toxin (DT) into $C X_{3} C R 1-i D T R$ mice [40]. Twomonth-old $C X_{3} C R 1$-iDTR mice first received $10 \mathrm{mg}$ of tamoxifen by gavage both on Day 1 and Day 3 to induce Cre-mediated recombination and DTR expression under $C X_{3} C R 1$ promoter. $1 \mu \mathrm{g}$ DT was then administrated by intraperitoneal injections for three consecutive days on Day 10-12. The effect of DT administration on microglia depletion was examined 1 to 14 days after DT administration (Fig. 1a). We found that $C X_{3} C R 1-i D T R$ mice had a marked reduction of microglia 1 day after DT 


\section{a}

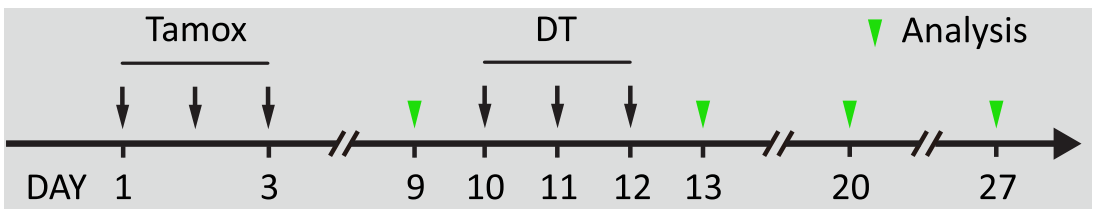

b

D9
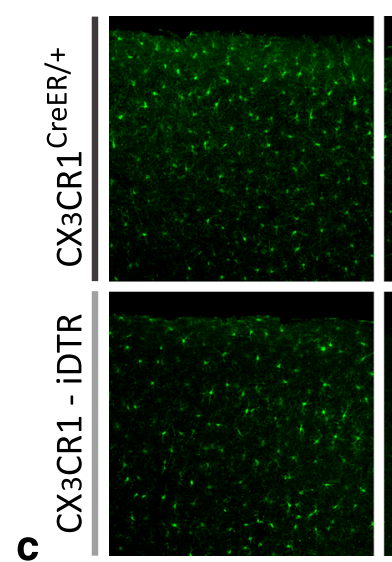

C
D13
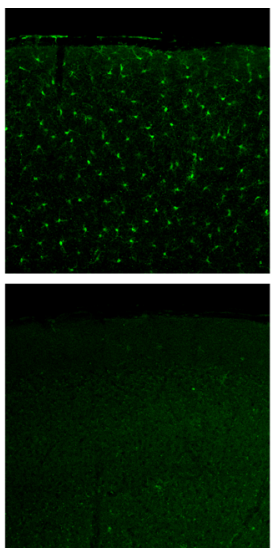

D20
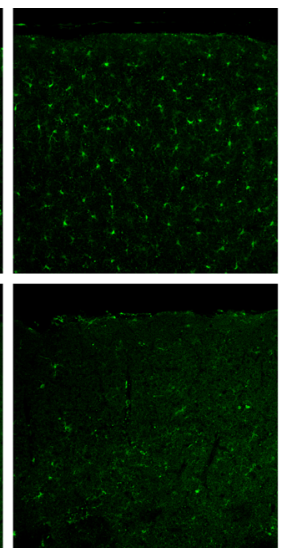

D27
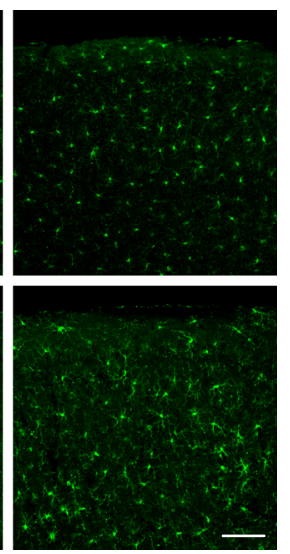

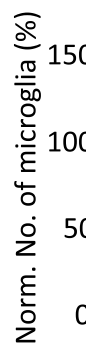

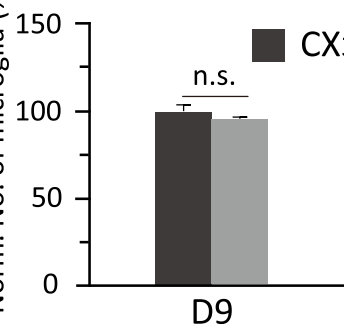

$\mathrm{CX} 3 \mathrm{CR} 1 \mathrm{CreER/+}$

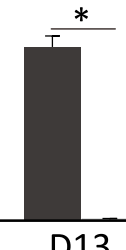

CX3CR1 - iDTR

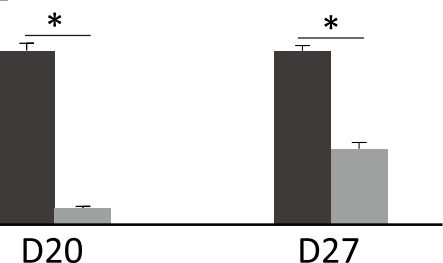

Fig. 1 Microglia are depleted in $C X_{3} C R 1$-iDTR mice over 1-7 days after DT administration and repopulate within 2 weeks after DT administration. a. Timeline of tamoxifen administration, DT administration and analysis of microglia number. Microglia were examined by Iba1 staining before (D9), 1 day after (D13), 1 week after (D20) and 2 weeks after DT administration (D27). b. Coronal sections of the cortex stained for Iba1 from $C X_{3} C R 1^{\text {CreER/+ }}$ and $C X_{3} C R 1-i D T R$ mice on D9, D13, D20 and D27, respectively. Scale bar, $100 \mu m$. c. Quantification of Iba1 positive cells showing that the majority of microglia were depleted in the first week after DT administration and $\sim 50 \%$ of microglia were repopulated during the second week in $C X_{3} C R 1$-iDTR mice $(n=4, * P<0.05$, Mann-Whitney test)

administration (Day 13) and the surviving microglia number was $0.8 \pm 0.8 \%$ compared with control mice without DTR expression. Microglia remained largely absent in the cortex 7 days after DT administration (Day $20,13.1 \pm 1.3 \%) .2$ weeks after DT administration, $57.4 \pm 4.6 \%$ of microglia were found in the cortex of $C X_{3} C R 1-i D T R$ mice, indicating microglia repopulation occurred at this stage (Fig. 1b, c). Similar dynamics of microglia depletion and repopulation following DT administration were observed in the cortex of APP/PS1/ $C X_{3} C R 1-i D T R$ mice at 15 month of age (Additional file 1: Figure S1). Thus, consistent with previous findings [40], these results show that after DT administration, microglia are efficiently depleted from the cortex of $C X_{3} C R 1-i D T R$ mice or $A P P / P S 1 / C X_{3} C R 1-i D T R$ mice within one week, but start to repopulate during the second week.

\section{Microglia depletion over 2 weeks has no effect on the} number of plaques

To investigate whether microglia depletion affects $A \beta$ deposition, we crossed $A P P / P S 1$ mice with $C X_{3} C R 1-i D T R$ mice and depleted microglia in mice more than 12 months old (17 \pm 2 months old). We first examined the effect of microglia depletion on the number of amyloid plaques over 1-2 weeks after DT administration (Fig. 2a). To label A $\beta$ plaques, Congo Red was injected into the subarachnoid space 24-48 h before each imaging session (see Methods). Congo Red-labeled $A \beta$ plaques were imaged through thinned-skull window using two-photon microscopy. Time-lapse imaging of the same cortical regions was performed 1 day (Day 13), 1 week (Day 20) and 2 weeks (Day 27) after DT administration (Fig. 2b, d). We found that the average plaque density in $A P P / P S 1 / C X_{3} C R 1-i D T R$ mice before DT administration was $515 \pm 63 \mathrm{~mm}^{-3}$, 


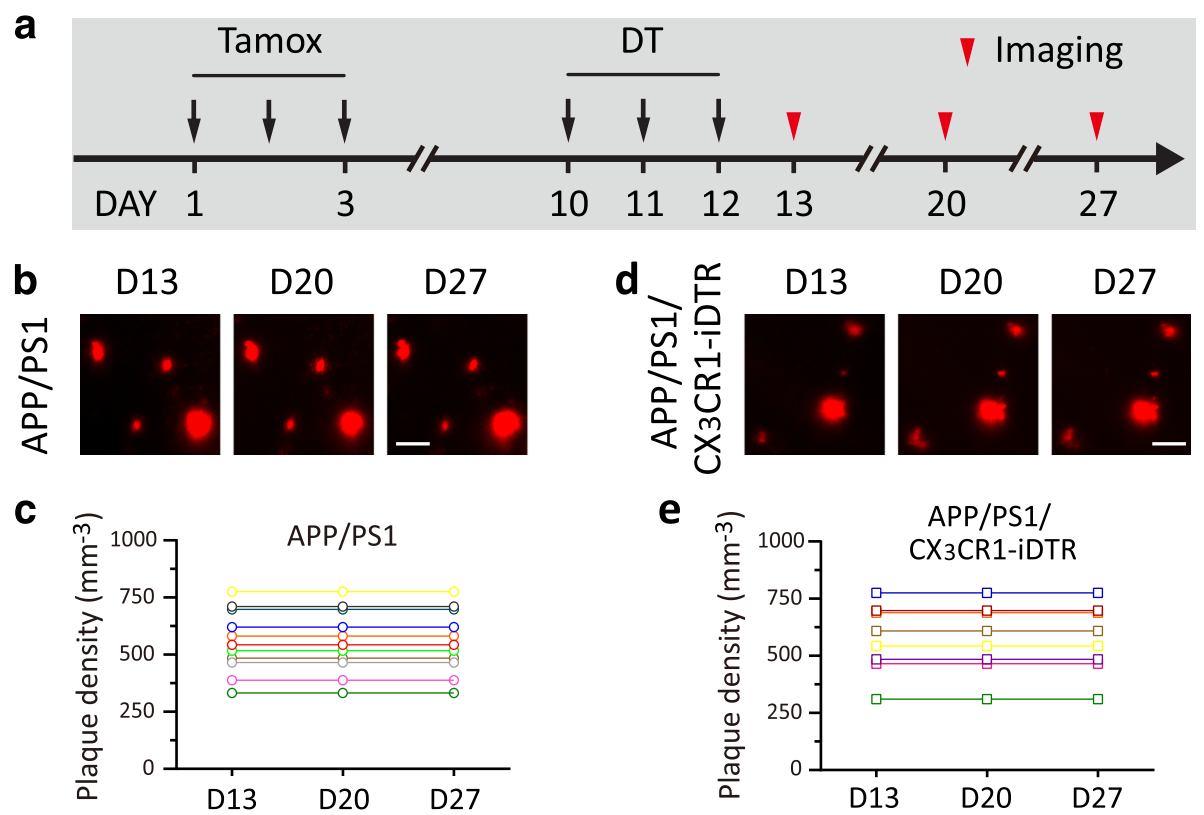

Fig. 2 Ablation of microglia by DT administration has no effect on the number of amyloid plaques. a. Timeline of tamoxifen administration, DT administration and in vivo imaging of Congo Red-labeled plaques. Time-lapse imaging was performed 1 day after (D13), 1 week after (D20) and 2 weeks after DT administration (D27). b. Images of the same ROls in APP/PS1 mice over 1-2 weeks. c. Quantification of plaque density in APP/PS1 mice in each ROI. d. Images of the same ROls in APP/PS1/CX ${ }_{3} C R 1$-iDTR mice over 1-2 weeks. Neither elimination of existing A $\beta$ plaques nor formation of new plaques occurred in APP/PS1 or APP/PS1/CX 3 CR1-iDTR mice. e. Quantification of plaque density in APP/PS1/CX ${ }_{3} C R 1$-iDTR mice in each ROI

comparable to that in APP/PS1 mice $\left(574 \pm 42 \mathrm{~mm}^{-3}\right.$, $P>0.05)$. Consistent with the previous study showing no formation of new plaques over weeks in APP/PS1 mice older than 9 months [42], we found that in >12 monthold $A P P / P S 1$ mice, no disappearance of existing plaques or appearance of new plaques was detected over 1-2 weeks (Fig. 2b, c). Notably, the number of $A \beta$ plaques also remained the same over $1-2$ weeks in mice when microglia were depleted by DT administration (Fig. 2d, e). No new plaques or disappearance of existing plaques was observed during this period. Thus, consistent with the previous study reporting microglia depletion has no significant effect on the number of plaques [38, 39], our findings suggest that ablation of microglia by DT administration does not affect the number of $A \beta$ plaques over $1-2$ weeks.

\section{Microglia depletion leads to enlargement of $A \beta$ plaques} In addition to examining the effect of microglia depletion on plaque number, we also compared the size of plaques over time in mice with or without microglia by two-photon time-lapse imaging. The Congo Red-labeled area of plaque was used as the measurements of plaque size. The border of amyloid plaques was determined such that the fluorescence intensity of amyloid plaques was above 3 times of standard deviation of the background fluorescence (Fig. 3a, see Methods). The size of $\mathrm{A} \beta$ plaques identified in APP/PS1 $\left(308.9 \pm 27.6 \mu \mathrm{m}^{2}\right)$ and $A P P / P S 1 / C X_{3} C R 1-i D T R$ mice $\left(343.3 \pm 35.5 \mu \mathrm{m}^{2}\right)$ were comparable before DT administration $(P=0.23$, Fig. 3b). Consistent with previous studies [42-44], we found that the size of plaques showed no significant change over 1-2 weeks in APP/PS1 mice older than 12 months (Fig. 3c-f). In contrast to the stable size of amyloid plaques in $A P P / P S 1$ mice, we found that after DT administration plaques showed a significant increase $(12.8 \pm 3.2 \%, P<0.001)$ in size from Day 13 to Day 20 in $A P P / P S 1 / C X_{3} C R 1-i D T R$ mice (Fig. 3d, e). Furthermore, during the second week after DT administration when microglia repopulated (Fig. 1b, c), we found that $A P P / P S 1 / C X_{3} C R 1-i D T R$ mice showed no additional increase of plaque size from Day 20 to Day $27(P=0.32$, Fig. 3d, f). Taken together, these results strongly suggest that microglia have an important role in limiting the growth of amyloid plaques.

To further understand how the absence of microglia affects amyloid deposition, we examined Congo Red fluorescence intensity surrounding plaques. Fluorescence intensity across a radial line from plaque center towards outer area was measured (Fig. 4a) and separated into the "inside" part and the "outside" part by the border of plaque (Fig. 4b, see Methods). By examining fluorescence intensity within $5 \mu \mathrm{m}$ surrounding plaque border in the "outside" part, we found a significant increase of Congo Red fluorescence intensity from Day 13 to Day 20 (43.7\%, $P<0.001$, Fig. 4c). In the following second week when microglia repopulated (Day 20 to Day 27), 

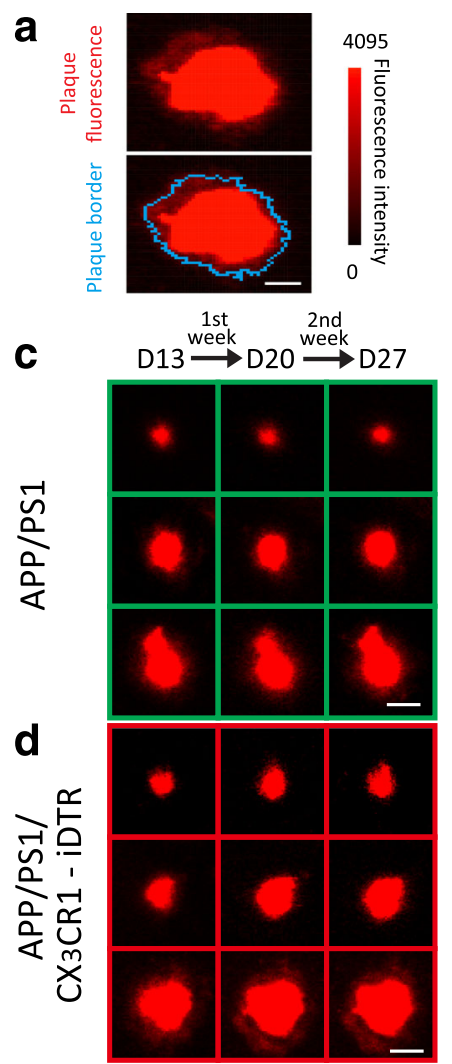

b

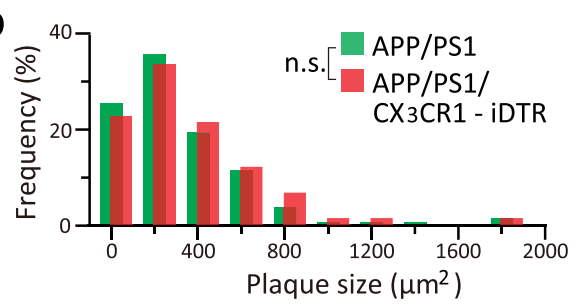

e

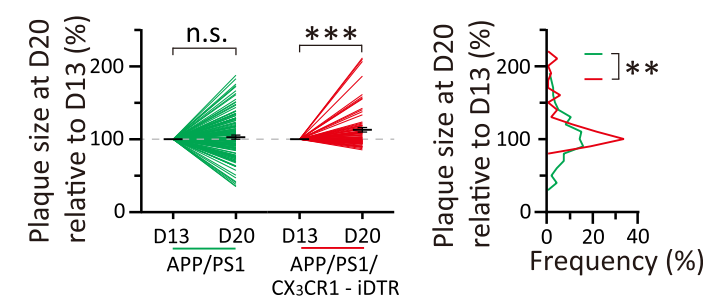

f

2nd week
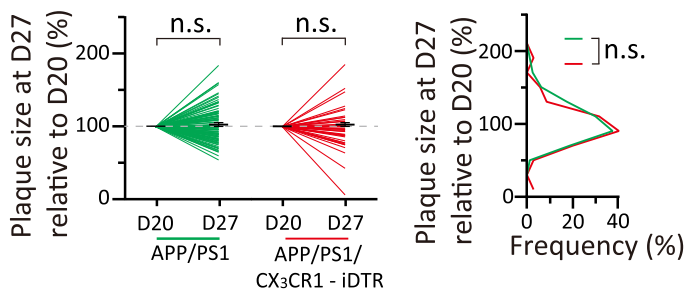

Fig. 3 Microglia depletion induces increase in plaque size. a. Blue curve in the bottom panel indicates the identified plaque border for the example in the upper panel. The border of amyloid plaque was determined such that the fluorescence intensity of amyloid plaques was above 3 times of s.d. $\mathbf{b}$. Distribution of plaque size on Day 13 in the cortex of APP/PS1 mice (green bars, $n=129$ plaques from 5 mice) and APP/PS1/CX ${ }_{3}$ CR1-iDTR mice (red bars, $n$ $=75$ plaques from 4 mice) $(P=0.23$, Mann-Whitney test). $\mathbf{c}$. Three examples of plaques imaged over 1-2 weeks in APP/PS1 mice. $\mathbf{d}$. Three examples of plaques imaged over 1-2 week in APP/PS1/CX 3 CRT-iDTR mice. e. Change of plaque size in the first week after DT administration. No significant change in plaque size was detected within one week in APP/PS1 mice ( $n=129$ plaques from 5 mice, $P=0.16$, Wilcoxon matched-pairs signed rank test). Microglia depletion induced a significant increase in plaque size in APP/PS1/CX $X_{3} C R 1$-iDTR mice $\left(n=75\right.$ plaques from 4 mice, ${ }^{* * *} P<0.001$, Wilcoxon matched-pairs signed rank test). The distribution of plaque size at D20 relative to D13 is significantly different between these two groups (right panel, ${ }^{* *} P<0.01$, MannWhitney test). $\mathbf{f}$. Change of plaque size in the second week after DT administration. No significant change in plaque size was detected in APP/PS1 mice ( $n=92$ plaques from 5 mice, $P=0.52$, Wilcoxon matched-pairs signed rank test). A $\beta$ plaques in APP/PS1/CX 3 CR1-iDTR mouse cortex didn't continue to grow from D20 to D27 ( $n=40$ plaques from 4 mice, $P=0.32$, Wilcoxon matched-pairs signed rank test). The distribution of plaque size at D27 relative to D20 is not significantly different between these two groups (right panel, $P=0.52$, Mann-Whitney test). Scale bar in a, c and d, $20 \mu \mathrm{m}$

fluorescence intensity in the surrounding area didn't continue to increase $(P=0.84$, Fig. $4 \mathrm{~d})$. The change and stabilization of Congo Red fluorescence surrounding plaques after microglia depletion and repopulation provide further evidence that microglia are involved in limiting plaque growth.

\section{$A \beta$ deposits cause abnormalities in dendrites passing through plaques}

Our findings in $A P P / P S 1 / C X_{3} C R 1-i D T R$ mice showed that microglia depletion resulted in $\sim 13 \%$ increase in plaque size within one week. Based on the averaged plaque size in APP/PS1 mice (308.9 $\pm 27.6 \mu \mathrm{m}^{2}$, Fig. $\left.3 \mathrm{~b}\right)$, we estimated that for each plaque the cortex area covered by $\mathrm{A} \beta$ accumulation increase by $\sim 40 \mu \mathrm{m}^{2}$ over one week in the absence of microglia. Previous studies have shown various abnormalities of axons and dendrites inside or near plaques [5, 7-10]. It is possible that $\sim 13 \%$ expansion of plaques after microglia depletion may lead to more extensive damage in neuronal circuits. To better understand neuronal damage associated with plaques, we examined spine density and shaft diameter of dendrites passing through and outside plaques in fixed brain slices. In this experiment, PS1 mutant mice $\left(P S 1_{\mathrm{M} 146 \mathrm{~L}}\right.$ line 6.2) were first crossed with $A P P$ mutant mice (FAD $A P P_{670 / 671}$ line $\left.\mathrm{Tg} 2576\right)$. This mouse model of $\mathrm{AD}$ (PSAPP mice) was further crossed with Thy1 YFP H-line mice to visualize dendrites and dendritic spines of pyramidal neurons in fixed brain slices from 6 to 10month-old mice. Amyloid plaques were labeled with Congo Red (Fig. 5a). When dendritic segments passing through plaques were divided into "proximal" segments 

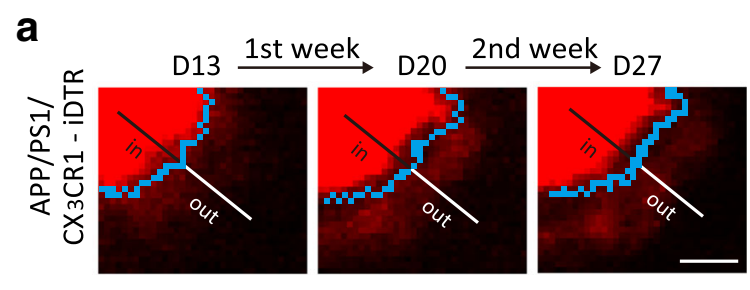

b

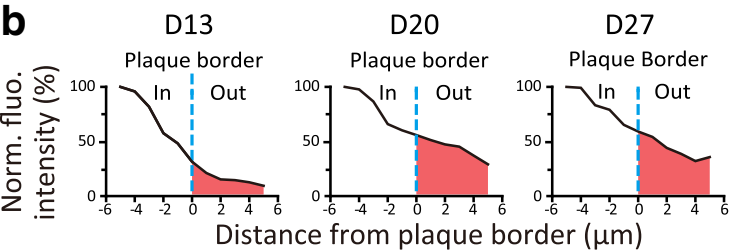

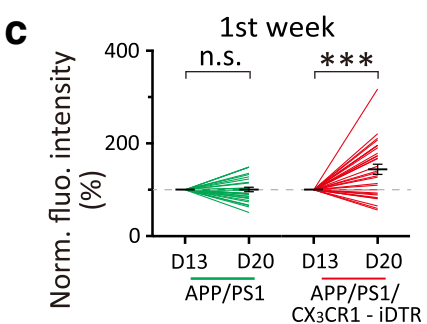

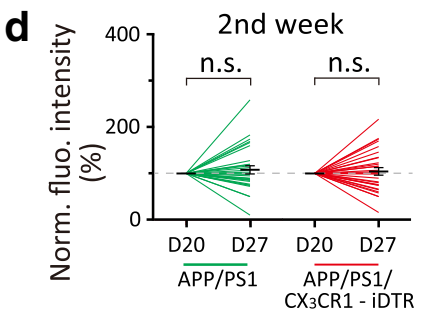

Fig. 4 Absence of microglia induces increase of Congo Red-labeled A fluorescence surrounding plaques. a. An example of an AB plaque in APP/PS1/ $C X_{3}$ CR1-iDTR mice at D13, D20 and D27, with blue curves indicating plaque border. A radial line from the center point towards the outer area of a plaque is separated by the plaque border, with the inside part representing area in the plaque ("in", black) and the outside part representing the area surrounding the plaque ("out", white). Scale bar, $5 \mu \mathrm{m}$. b. Fluorescence intensity across the line in a, with $5 \mu \mathrm{m}$ inside the plaque and $5 \mu \mathrm{m}$ outside the plaque. Shadow area represents fluorescence intensity surrounding plaques. c. Normalized fluorescence intensity surrounding plaques from D13 to D20. Microglia depletion in APP/PS1/CX 3 CR1-iDTR mice induced a significant increase of fluorescence in the area surrounding plaque $\left(n=30\right.$ plaques, ${ }^{* * *} P<0.001$, Wilcoxon matched-pairs signed rank test). $\mathbf{d}$. In the second week after DT administration (from D20 to D27), fluorescence surrounding plaques showed no additional increase in APP/PS1/CX 3 CR1-iDTR mice ( $n=30$ plaques, $P=0.84$, Wilcoxon matched-pairs signed rank test)
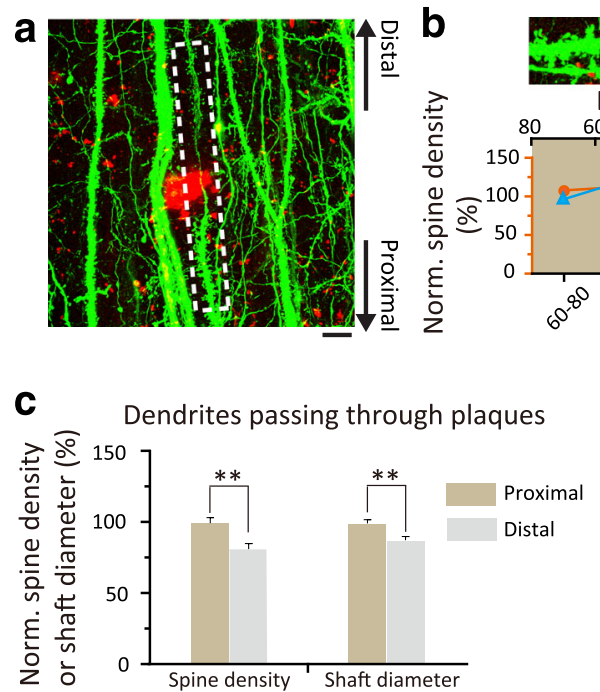
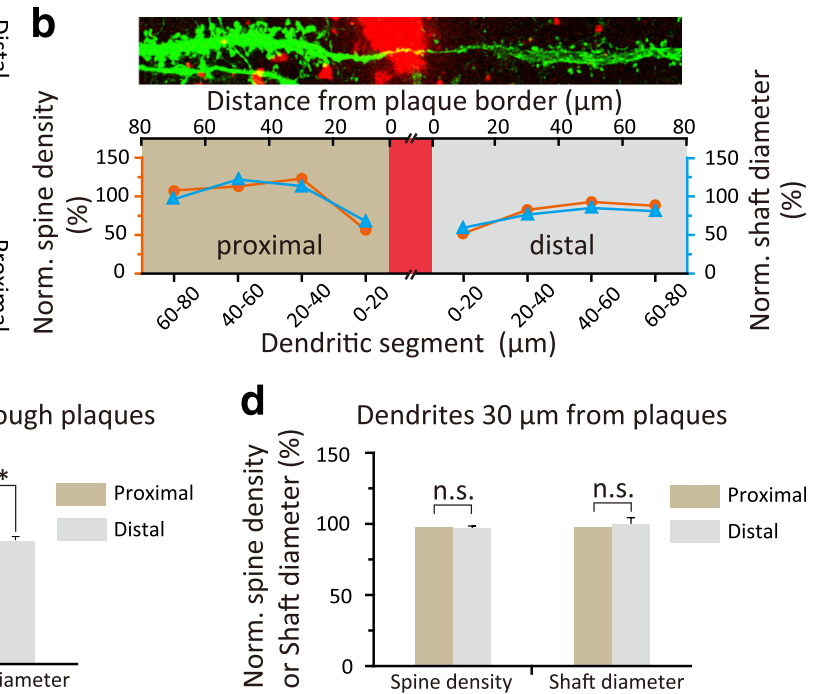

Fig. 5 A $\beta$ plaques cause spine loss and shaft atrophy in the distal segments of dendrites passing through plaques a. Confocal image of YFP labeled dendrites passing through and near A $\beta$ plaques in the fixed brain slice of PSAPP mice crossed with Thy 1 YFP H-line mice. A $\beta$ plaques were labeled with Congo red (red). A yellow fluorescent protein-labeled dendrite (green) showed a significant reduction in spine density and dendritic diameter after entering the plaque. Scale bar, $20 \mu \mathrm{m}$. b. Upper panel shows the dendritic branch passing through the plaque in dashed area in a. Bottom panel shows the quantification of spine density (orange curve) and shaft diameter (blue curve) in every $20 \mu \mathrm{m}$ long of dendritic segments, ranging from 0 to $80 \mu \mathrm{m}$ from plaque border. $\mathbf{c}$. Distal segments of dendrites passing through A $\beta$ plaques showed reduced spine density and shaft diameter compared to proximal segments. ( $n=22$ dendrites from 4 mice, ${ }^{* *} P<0.01$, student t-test). $\mathbf{d}$. Spine density and shaft diameter of dendrites located greater than $30 \mu \mathrm{m}$ away from plaque deposits showed no difference in the proximal and distal segments $(n=22$ dendrites from 4 mice, $P>0.05$, student t-test) 
(segments between the cell body and the plaque) and "distal" segments (remaining segments after exiting the plaque, Fig. 5a, b), we found that distal segments of dendrites passing through plaques showed $19.4 \pm 2.8 \%$ reduction in spine density and $11.1 \pm 1.3 \%$ reduction in shaft diameter $(P<0.01)$ as compared to proximal segments (Fig. 5c). In contrast, dendrites that did not pass through plaques and were located $>30 \mu \mathrm{m}$ away from plaque border showed no significant difference in spine density and shaft diameter between the corresponding distal and proximal segments (Fig. 5d). These findings show that amyloid plaque formation not only leads to dendritic abnormalities inside plaques, but also causes more global changes in distal segments of dendrites passing through plaques. The alterations of distal parts of dendrites passing through plaques suggest that $~ 13 \%$ expansion of amyloid plaques after microglia depletion could cause sizable damage in neuronal connectivity.

\section{Discussion}

It is generally believed that microglia are involved in regulating $A \beta$ deposition, a key hallmark of Alzheimer's disease. However, the precise role of microglia in plaque formation and maintenance remains unclear. There are conflicting views on whether microglia are critical for limiting $\mathrm{A} \beta$ plaques, or microglia have no effect on plaque deposition. In this work, we examined the function of microglia in $\mathrm{A} \beta$ deposition by depleting microglia and imaging $A \beta$ plaques in the living cortex of an APP/PS1 mouse model. Our findings indicate the size of amyloid plaques increases in the absence of microglia. We further show that $\mathrm{A} \beta$ plaques are associated with dendritic spine loss and shaft atrophy in dendrites passing through plaques. These findings suggest that microglia play an important role in limiting $A \beta$ plaques growth and neuronal damage in Alzheimer's disease.

\section{Microglia limit the expansion of $A \beta$ plaques}

Mutations of CD33 and TREM2 genes expressed in microglia have been linked to increased risks of $\mathrm{AD}[26,27,37]$. Altered $\mathrm{A} \beta$ load has been observed in $C X_{3} C R 1^{-/-}[29,30]$, TREM2 $^{-{ }^{--}}$and $C D 33^{-/-}$mice [27]. In vitro studies have shown that microglia phagocytose $A \beta$ [33-35]. Furthermore, in vivo studies have shown that $A \beta$ is localized within microglial lysosomes and microglia volume surrounding plaques correlates with the reduction of plaque size over one month [25]. Together, these studies strongly suggest a role of microglia in $A \beta$ clearance and/or regulating $d A \beta$ deposition. However, previous studies of depleting microglia by introducing suicide gene HSVTK or inhibiting CSFR 1 have shown that microglia depletion has no effect on both formation and maintenance of plaques $[38,39]$. In the present study, using Cre-dependent microglia depletion and time-lapse imaging, we have now provided direct evidence that in the $\mathrm{AD}$ mouse model older than 12 months, microglia depletion over 1 week does not affect the formation or maintenance of amyloid plaques, but leads to a 13\% enlargement of plaque size in the cortex (Fig. 3d, e). Furthermore, 2 weeks after DT administration, microglia repopulation was associated with the stabilization of plaque size (Fig. 3d, f). Our findings strongly suggest the role of microglia in restricting the expansion of plaques.

It is important to point out several differences between previous studies and our work on the effect of microglia depletion. One difference is that previous studies examined the number and size of amyloid plaques using fixed brain tissues from different animals with or without microglia depletion $[38,39]$. The variability in plaque number and size between different animals may make it difficult to detect relatively small changes in plaque size after microglia depletion. Taking advantage of time-lapse imaging, we have been able to track the same plaques over time and reveal the relative small changes $(\sim 13 \%$ over one week) of plaque size in response to microglia depletion. It is also important to note that microglia depletion in previous studies may take longer to occur than in our studies. The prolonged process of microglia depletion might cause compensatory responses such as astrocyte activation, which could lead to degradation of $A \beta[45,46]$. Further studies are needed to address these possibilities in order to better understand the role of microglia in amyloid plaque deposition.

In addition to microglia in the brain, peripheral myeloid cells also express $\mathrm{CX}_{3} \mathrm{CR} 1$ and therefore could be depleted by DT administration in $A P P / P S 1 / C X_{3} C R 1-i D T R$ mice. However, microglia and peripheral $\mathrm{CX}_{3} \mathrm{CR} 1^{+}$cells have substantially different turnover rates and are derived from different precursor populations [40, 47-50]. Microglia are long-lived population [47] and it has been shown that when tamoxifen is administrated $\sim 30$ days prior to the administration of DT, $C X_{3} C R 1-i D T R$ mice have a dramatic reduction of microglia within 1 day after DT administration [40]. On the other hand, $\mathrm{CX}_{3} \mathrm{CR}^{+}{ }^{+}$cells in the spleen or blood are not affected as these peripheral $\mathrm{CX}_{3} \mathrm{CR}^{+}$ myeloid cells in $C X_{3} C R 1-i D T R$ mice are replenished through a $\mathrm{CX}_{3} \mathrm{CR}^{-}$bone marrow precursor [47-50] and no longer express DT receptors 30 days after tamoxifen administration [40]. In our experiment, the time period between tamoxifen and DT administration was 7 days. Based on the rapid turnover of peripheral $\mathrm{CX}_{3} \mathrm{CR} 1^{+}$cells (Fig. 2 in ref. [40]), we expect that over this 7 day period the majority of peripheral $\mathrm{CX}_{3} \mathrm{CR} 1^{+}$cells would be replenished through a $\mathrm{CX}_{3} \mathrm{CR} 1^{-}$bone marrow precursor and do not express DT receptors. A small fraction of peripheral myeloid cells would still express DT receptors and would be depleted following DT administration. Therefore, we could not completely rule out the possibility that in addition to microglia depletion, the depletion of some 
peripheral $\mathrm{CX}_{3} \mathrm{CR}^{+}$myeloid cells may also affect the growth of amyloid plaques. Future studies to specifically deplete peripheral $\mathrm{CX}_{3} \mathrm{CR} 1^{+}$myeloid cells using bone marrow transplantation are needed to determine the potential contribution of peripheral $\mathrm{CX}_{3} \mathrm{CR} 1^{+}$cell population in amyloid plaque formation.

\section{Plaque growth may cause extensive neurite damage}

A variety of studies have shown that abnormal spine density, spine turnover and diameter of dendritic shaft are associated with $A \beta$ plaques $[5,7-9,51]$. In the current study, we found dendritic spine loss and shaft atrophy in the distal segments of dendrites passing through plaques when compared with proximal segments in the PSAPP mouse model of $\mathrm{AD}$ (Fig. $5 \mathrm{a}-\mathrm{c}$ ). This distal effect was not found in the dendrites which did not pass through plaques and were $30 \mu \mathrm{m}$ away from $A \beta$ plaques (Fig $5 \mathrm{~d}$ ). These results suggest that the expansion of $A \beta$ plaques could cause damage not only in the local position but also to the entire distal segments of dendrites passing through. In this way, amyloid deposits might disrupt the signal propagation and protein transportation generated from soma and further lead to the degeneration of distal segments. The distal effect in dendrites passing through plaques also implies that extensive damage of neurites could be caused by enlargement of $A \beta$ plaques when microglia are absent or microglia function is altered. These findings underscore the role of microglia in restricting the enlargement of plaques and limiting the neuronal damage in Alzheimer's disease. The expansion of amyloid plaques after microglia depletion and plaque-associated dendritic abnormalities likely contribute to memory loss and cognitive decline in AD [15-19]. However, it is worth mentioning that microglia in $\mathrm{AD}$ mice also likely have impacts on the function of neuronal circuits and animal's behaviors beyond their role in amyloid deposition and associated dystrophic neuritis $[40,52]$. Thus, the depletion of microglia and resulting plaque expansion may or may not indicate disease worsening in $\mathrm{AD}$ mice. Future studies are needed to examine the impact of microglia depletion on neuronal network activity and plaqueassociated synaptic abnormalities in the living mouse cortex in order to better understand microglia functions in $\mathrm{AD}$. Furthermore, while plaque-associated dystrophic neurites have been shown in several $\mathrm{AD}$ mouse models and $\mathrm{AD}$ patients $[4,5,7,9,51,53]$, whether similar effects of amyloid plaques on distal dendrites observed in PSAPP mice also occur in other mouse models of $\mathrm{AD}$ remains to be investigated.

\section{Conclusion}

Our data highlight that microglia play an important role in limiting the growth of $A \beta$ plaques. Microglia depletion does not affect plaque numbers, but causes a significant increase of plaque size over one week. Furthermore, microglia repopulation is associated with the stabilization of plaque size. Restricting the growth of plaques by microglia could have a significant impact on reducing abnormalities in dendrites passing through amyloid plaques. Taken together, our studies reveal an important role of microglia in limiting the growth of $A \beta$ deposition and neuronal damage in the pathogenesis of $\mathrm{AD}$.

\section{Additional file}

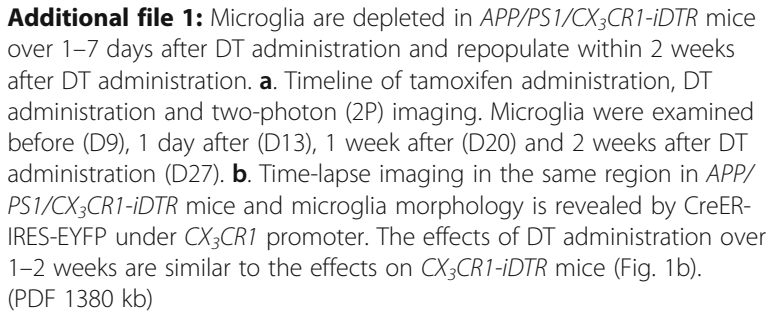

Additional file 1: Microglia are depleted in APP/PS1/CX ${ }_{3} C R 1-i D T R$ mice over 1-7 days after DT administration and repopulate within 2 weeks after DT administration. a. Timeline of tamoxifen administration, DT administration and two-photon (2P) imaging. Microglia were examined before (D9), 1 day after (D13), 1 week after (D20) and 2 weeks after DT administration (D27). b. Time-lapse imaging in the same region in APP/ PS1/CX ${ }_{3} C R 1$-iDTR mice and microglia morphology is revealed by CreERIRES-EYFP under $C X_{3} C R 1$ promoter. The effects of DT administration over $1-2$ weeks are similar to the effects on $C X_{3} C R 1$-iDTR mice (Fig. 1b). (PDF $1380 \mathrm{~kb}$ )

\section{Abbreviations \\ ACSF: artificial cerebral spinal fluid; AD: Alzheimer's disease; APP/PS1: APPswe/ PSEN1dE9; $A \beta$ : $\beta$-amyloid; $\mathrm{CX}_{3} \mathrm{CR} 1$-iDTR: $\mathrm{CX}_{3} \mathrm{CR}^{\mathrm{CreER} /+}: \mathrm{R}^{\mathrm{D} 6^{\mathrm{DTR} /+}}$; DT: diphtheria toxin; DTR: diphtheria toxin receptor; PSAPP: PS1 ${ }_{\text {M146L }}$ line $6.2 \times \operatorname{Tg}_{2576}$; $\mathrm{ROI}$ : region of interest}

\section{Acknowledgement}

We thank members of Gan lab for critical comments and helpful discussions on this manuscript.

\section{Funding}

This work was supported by NIH 5R01 NS087198 and the Investigator Initiated Research Grant from Alzheimer's Association (IIRG-08-92101) to W.-B. G., Shenzhen Science and Technology Innovation Funds KQTD2015032709315529, JSGG20140703163838793, JSYJ20160428154351820 and ZDSYS201504301539161 to W. L.

\section{Availability of data and materials}

All data analyzed during this study are included in this published article and additional files; images available from the corresponding author on reasonable request.

\section{Authors' contributions}

RZ, WL and WG designed the experiments and wrote the manuscript; RZ and $\mathrm{WL}$ performed in vivo imaging; JT performed confocal imaging; $\mathrm{WH}$ bred quadruple transgenic mice and performed genotyping; RZ and JT analyzed the data; All authors read and approved the final manuscript.

\section{Authors' information}

Information for all the co-authors is listed in the title page.

\section{Competing interests}

The authors declare that they have no competing interests.

\section{Consent for publication}

Not applicable.

\section{Ethics approval}

Animal studies with APP/PS1 mice and APP/PS1/CX ${ }_{3} C R 1$-iDTR mice were performed in compliance with the institutional guidelines at Peking University Shenzhen Graduate School. Animal studies with APP $670 / 671$ line Tg2576 mice and PS1 ${ }_{\text {M146L }}$ line 6.2 mice were performed in compliance with IACUC (Institutional Animal Care and Use Committee) at New York University School of Medicine. 


\section{Publisher's Note}

Springer Nature remains neutral with regard to jurisdictional claims in published maps and institutional affiliations.

\section{Author details}

'Drug Discovery Center, Peking University Shenzhen Graduate School, Shenzhen 518055, China. ${ }^{2}$ Skirball Institute, New York University School of Medicine, New York, NY 10016, USA.

Received: 24 December 2016 Accepted: 2 June 2017

\section{Published online: 12 June 2017}

\section{References}

1. Querfurth HW, LaFerla FM. Alzheimer's disease. N Engl J Med. 2010;362:329-44.

2. Scheltens $P$, Blennow K, Breteler MM, de Strooper B, Frisoni GB, Salloway S, et al. Alzheimer's disease. Lancet. 2016;388:505-17.

3. Cummings BJ, Su JH, Geddes JW, Van Nostrand WE, Wagner SL, Cunningham DD, et al. Aggregation of the amyloid precursor protein within degenerating neurons and dystrophic neurites in Alzheimer's disease. Neuroscience. 1992;48:763-77.

4. Knowles RB, Wyart C, Buldyrev SV, Cruz L, Urbanc B, Hasselmo ME, et al. Plaque-induced neurite abnormalities: implications for disruption of neural networks in Alzheimer's disease. Proc Natl Acad Sci U S A. 1999:96:5274-9.

5. Grutzendler J, Helmin K, Tsai J, Gan WB. Various dendritic abnormalities are associated with fibrillar amyloid deposits in Alzheimer's disease. Ann N Y Acad Sci. 2007;1097:30-9.

6. Serrano-Pozo A, Betensky RA, Frosch MP, Hyman BT. Plaque-associated local toxicity increases over the clinical course of Alzheimer disease. Am J Pathol. 2016;186:375-84.

7. Tsai J, Grutzendler J, Duff K, Gan WB. Fibrillar amyloid deposition leads to local synaptic abnormalities and breakage of neuronal branches. Nat Neurosci. 2004;:11181-3

8. Adalbert R, Nogradi A, Babetto E, Janeckova L, Walker SA, Kerschensteiner M, et al. Severely dystrophic axons at amyloid plaques remain continuous and connected to viable cell bodies. Brain. 2009;132:402-16.

9. Spires TL, Meyer-Luehmann M, Stern EA, McLean PJ, Skoch J, Nguyen PT, et al. Dendritic spine abnormalities in amyloid precursor protein transgenic mice demonstrated by gene transfer and intravital multiphoton microscopy. J Neurosci. 2005;25:7278-87.

10. Bittner T, Burgold S, Dorostkar MM, Fuhrmann M, Wegenast-Braun BM, Schmidt $B$, et al. Amyloid plaque formation precedes dendritic spine loss. Acta Neuropathol. 2012;124:797-807.

11. Walsh DM, Klyubin I, Fadeeva JV, Cullen WK, Anwyl R, Wolfe MS, et al. Naturally secreted oligomers of amyloid beta protein potently inhibit hippocampal long-term potentiation in vivo. Nature. 2002;416:535-9.

12. Akhtar MW, Sanz-Blasco S, Dolatabadi N, Parker J, Chon K, Lee MS, et al. Elevated glucose and oligomeric beta-amyloid disrupt synapses via a common pathway of aberrant protein S-nitrosylation. Nat Commun. 2016;7:10242.

13. Busche MA, Eichhoff $G$, Adelsberger $H$, Abramowski D, Wiederhold KH, Haass $C$, et al. Clusters of hyperactive neurons near amyloid plaques in a mouse model of Alzheimer's disease. Science. 2008;321:1686-9.

14. Busche MA, Kekus M, Adelsberger H, Noda T, Forstl H, Nelken I, Konnerth A. Rescue of long-range circuit dysfunction in Alzheimer's disease models. Nat Neurosci. 2015;18:1623-1630.

15. Terry RD, Masliah E, Salmon DP, Butters N, DeTeresa R, Hill R, et al. Physical basis of cognitive alterations in Alzheimer's disease: synapse loss is the major correlate of cognitive impairment. Ann Neurol. 1991;30:572-80.

16. Masliah E, Ellisman M, Carragher B, Mallory M, Young S, Hansen L, et al. Three-dimensional analysis of the relationship between synaptic pathology and neuropil threads in Alzheimer disease. J Neuropathol Exp Neurol. 1992:51:404-14.

17. Spires-Jones TL, Hyman BT. The intersection of amyloid beta and tau at synapses in Alzheimer's disease. Neuron. 2014;82:756-71.

18. Hardy JA, Higgins GA. Alzheimer's disease: the amyloid cascade hypothesis. Science. 1992;256:184-5.

19. Jack CR Jr, Lowe VJ, Weigand SD, Wiste HJ, Senjem ML, Knopman DS, et al. Serial PIB and MRI in normal, mild cognitive impairment and Alzheimer's disease: implications for sequence of pathological events in Alzheimer's disease. Brain. 2009;132:1355-65.

20. Prinz M, Priller J, Sisodia SS, Ransohoff RM. Heterogeneity of CNS myeloid cells and their roles in neurodegeneration. Nat Neurosci. 2011;14:1227-35.
21. Gomez Perdiguero E, Schulz C, Geissmann F. Development and homeostasis of "resident" myeloid cells: the case of the microglia. Glia. 2013;61:112-20.

22. Rozemuller JM, Eikelenboom P, Stam FC. Role of microglia in plaque formation in senile dementia of the Alzheimer type. An immunohistochemical study. Virchows Arch B Cell Pathol Incl Mol Pathol. 1986;51:247-54.

23. Mattiace $L A$, Davies $P$, Yen $S H$, Dickson DW. Microglia in cerebellar plaques in Alzheimer's disease. Acta Neuropathol. 1990;80:493-8.

24. Meyer-Luehmann M, Spires-Jones TL, Prada C, Garcia-Alloza M, de Calignon A, Rozkalne A, et al. Rapid appearance and local toxicity of amyloid-beta plaques in a mouse model of Alzheimer's disease. Nature. 2008;451:720-4.

25. Bolmont T, Haiss F, Eicke D, Radde R, Mathis CA, Klunk WE, et al. Dynamics of the microglial/amyloid interaction indicate a role in plaque maintenance. J Neurosci. 2008;28:4283-92.

26. Hollingworth P, Harold D, Sims R, Gerrish A, Lambert JC, Carrasquillo MM, et al. Common variants at ABCA7, MS4A6A/MS4A4E, EPHA1, CD33 and CD2AP are associated with Alzheimer's disease. Nat Genet. 2011;43:429-35.

27. Griciuc A, Serrano-Pozo A, Parrado AR, Lesinski AN, Asselin CN, Mullin K, et al. Alzheimer's disease risk gene CD33 inhibits microglial uptake of amyloid beta. Neuron. 2013;78:631-43.

28. Jonsson T, Stefansson H, Steinberg S, Jonsdottir I, Jonsson PV, Snaedal J, et al. Variant of TREM2 associated with the risk of Alzheimer's disease. N Engl J Med. 2013;368:107-16.

29. Lee S, Varvel NH, Konerth ME, Xu G, Cardona AE, Ransohoff RM, et al. CX3CR1 deficiency alters microglial activation and reduces beta-amyloid deposition in two Alzheimer's disease mouse models. Am J Pathol. 2010;177:2549-62.

30. Liu Z, Condello C, Schain A, Harb R, Grutzendler J. CX3CR1 in microglia regulates brain amyloid deposition through selective protofibrillar amyloidbeta phagocytosis. J Neurosci. 2010;30:17091-101.

31. Wang Y, Cella M, Mallinson K, Ulrich JD, Young KL, Robinette ML, et al. TREM2 lipid sensing sustains the microglial response in an Alzheimer's disease model. Cell. 2015;160:1061-71.

32. Wang Y, Ulland TK, Ulrich JD, Song W, Tzaferis JA, Hole JT, et al. TREM2mediated early microglial response limits diffusion and toxicity of amyloid plaques. J Exp Med. 2016;213:667-75.

33. Paresce DM, Ghosh RN, Maxfield FR. Microglial cells internalize aggregates of the Alzheimer's disease amyloid beta-protein via a scavenger receptor. Neuron. 1996;17:553-65.

34. Koenigsknecht J, Landreth G. Microglial phagocytosis of fibrillar beta-amyloid through a beta1 integrin-dependent mechanism. J Neurosci. 2004;24:9838-46.

35. Herber DL, Mercer M, Roth LM, Symmonds K, Maloney J, Wilson N, et al. Microglial activation is required for Abeta clearance after intracranial injection of lipopolysaccharide in APP transgenic mice. J Neuroimmune Pharmacol. 2007;2:222-31.

36. Condello C, Yuan P, Schain A, Grutzendler J. Microglia constitute a barrier that prevents neurotoxic protofibrillar Abeta42 hotspots around plaques. Nat Commun. 2015;6:6176.

37. Yuan P, Condello C, Keene CD, Wang Y, Bird TD, Paul SM, et al. TREM2 Haplodeficiency in mice and humans impairs the microglia barrier function leading to decreased Amyloid compaction and severe axonal dystrophy. Neuron. 2016;90:724-39.

38. Grathwohl SA, Kalin RE, Bolmont T, Prokop S, Winkelmann G, Kaeser SA, et al. Formation and maintenance of Alzheimer's disease beta-amyloid plaques in the absence of microglia. Nat Neurosci. 2009;12:1361-3.

39. Spangenberg EE, Lee RJ, Najafi AR, Rice RA, Elmore MR, Blurton-Jones M, et al. Eliminating microglia in Alzheimer's mice prevents neuronal loss without modulating amyloid-beta pathology. Brain. 2016;139:1265-81.

40. Parkhurst CN, Yang G, Ninan I, Savas JN, Yates JR 3rd, Lafaille JJ, et al. Microglia promote learning-dependent synapse formation through brainderived neurotrophic factor. Cell. 2013;155:1596-609.

41. Yang G, Pan F, Parkhurst CN, Grutzendler J, Gan WB. Thinned-skull cranial window technique for long-term imaging of the cortex in live mice. Nat Protoc. 2010;5:201-8.

42. Hefendehl JK, Wegenast-Braun BM, Liebig C, Eicke D, Milford D, Calhoun ME, et al. Long-term in vivo imaging of beta-amyloid plaque appearance and growth in a mouse model of cerebral beta-amyloidosis. J Neurosci. 2011;31:624-9.

43. Yan P, Bero AW, Cirrito JR, Xiao Q, Hu X, Wang Y, et al. Characterizing the appearance and growth of amyloid plaques in APP/PS1 mice. J Neurosci. 2009;29:10706-14.

44. Burgold S, Bittner T, Dorostkar MM, Kieser D, Fuhrmann M, Mitteregger G, et al. In vivo multiphoton imaging reveals gradual growth of newborn amyloid plaques over weeks. Acta Neuropathol. 2011;121:327-35. 
45. Wyss-Coray T, Loike JD, Brionne TC, Lu E, Anankov R, Yan F, et al. Adult mouse astrocytes degrade amyloid-beta in vitro and in situ. Nat Med. 2003;9:453-7.

46. Koistinaho M, Lin S, Wu X, Esterman M, Koger D, Hanson J, et al. Apolipoprotein E promotes astrocyte colocalization and degradation of deposited amyloid-beta peptides. Nat Med. 2004;10:719-26.

47. Ajami B, Bennett JL, Krieger C, Tetzlaff W, Rossi FM. Local self-renewal can sustain CNS microglia maintenance and function throughout adult life. Nat Neurosci. 2007;10:1538-43.

48. Lawson LJ, Perry VH, Gordon S. Turnover of resident microglia in the normal adult mouse brain. Neuroscience. 1992;48:405-15.

49. van Furth $\mathrm{R}$, Cohn ZA. The origin and kinetics of mononuclear phagocytes. J Exp Med. 1968;128:415-35.

50. Fogg DK, Sibon C, Miled C, Jung S, Aucouturier P, Littman DR, et al. A clonogenic bone marrow progenitor specific for macrophages and dendritic cells. Science. 2006:311:83-7.

51. Kirkwood CM, Ciuchta J, Ikonomovic MD, Fish KN, Abrahamson EE, Murray PS, et al. Dendritic spine density, morphology, and fibrillar actin content surrounding amyloid-beta plaques in a mouse model of amyloid-beta deposition. J Neuropathol Exp Neurol. 2013;72:791-800.

52. Miyamoto A, Wake H, Ishikawa AW, Eto K, Shibata K, Murakoshi H, et al. Microglia contact induces synapse formation in developing somatosensory cortex. Nat Commun. 2016;7:12540.

53. Bittner T, Fuhrmann M, Burgold S, Ochs SM, Hoffmann N, Mitteregger G, et al. Multiple events lead to dendritic spine loss in triple transgenic Alzheimer's disease mice. PLoS One. 2010;5:e15477.

\section{Submit your next manuscript to BioMed Central and we will help you at every step:}

- We accept pre-submission inquiries

- Our selector tool helps you to find the most relevant journal

- We provide round the clock customer support

- Convenient online submission

- Thorough peer review

- Inclusion in PubMed and all major indexing services

- Maximum visibility for your research

Submit your manuscript at www.biomedcentral.com/submit

C) Biomed Central 